\title{
La rhétorique « Kleiz Breizh »
}

'Kleiz Breizh' rhetoric

\section{Mannaig Thomas}

\section{(2) OpenEdition}

\section{Journals}

Édition électronique

URL : https://journals.openedition.org/lbl/1897

DOI : $10.4000 / \mathrm{lbl} .1897$

ISSN : 2727-9383

\section{Éditeur}

Université de Bretagne Occidentale - UBO

\section{Édition imprimée}

Date de publication : 1 février 2013

Pagination : 181-201

ISBN : 979-10-92331-00-4

ISSN : 1270-2412

\section{Référence électronique}

Mannaig Thomas, « La rhétorique « Kleiz Breizh » », La Bretagne Linguistique [En ligne], 17 | 2013, mis en ligne le 01 mai 2021, consulté le 22 mai 2021. URL : http://journals.openedition.org//bl/1897 ; DOI : https://doi.org/10.4000/lbl.1897

\section{cc) (7)}

La Bretagne Linguistique est mise à disposition selon les termes de la Licence Creative Commons Attribution 4.0 International. 
Mannaig THOMAS*

\section{La rhétorique «Kleiz Breizh»}

\section{$\mathrm{L}$}

a rhétorique se voit parfois limitée à une liste des tropes ou de procédés rhétoriques laissant penser à une sorte de boîte à outils dans lesquels les uns - les auteurs - viennent piocher des figures dont ils supposent qu'elles vont être identifiées par les autres - les lecteurs - et constituer ainsi leur style. Si ce jeu a quelque chose de satisfaisant ou de ludique pour le critique, l'identification des figures n'est que le point de départ d'une véritable question d'analyse littéraire : qu'est-ce que l'emploi de tournures rhétoriques apporte au message que l'auteur souhaite délivrer au lecteur?

En 2003, Hervé Ar Gall ${ }^{1}$ publie Piv a glevo ? ${ }^{2}$ aux éditions An Alarc' $h^{3}$. Il s'agit d'un roman que l'on pourrait qualifier d'autofictif. C'est Serge Doubrovsky qui, le premier, a proposé, à la parution de son livre Fils ${ }^{4}$ en 1977, de qualifier son œuvre d'autofiction ${ }^{5}$,

* Maître de conférences de celtique, CRBC (EA 4451), UBO/ueb

1. Hervé Ar Gall est né à Loudéac en 1952.

2. Cf. Hervé Ar Gall, Piv a glevo ?, Lannion, An Alarc'h, 2003.

3. An Alarc'h est une maison d'édition d'ouvrages en langue bretonne. Créée en 2003, elle publie environ 4 ouvrages par an, essentiellement des romans ou des recueils de nouvelles.

4. Cf. Serge Doubrovsky, Fils, Paris, Galilée, 1977.

5. Sur la définition de l'autofiction, cf. Philippe GASPARINI, Autofiction. Une aventure du langage, Paris, Seuil, 2008. 
genre qu'il définit ainsi : "fiction d'événements et de faits strictement réels ${ }^{6} \gg$. L'apparition de ce terme peut être interprétée comme une manifestation de la suspicion qui entoure la sincérité du genre autobiographique ${ }^{7}$. L'autofiction joue, en effet, sur la proximité entre l'identité de l'auteur, du narrateur et du personnage principal, mais sans établir de réel pacte autobiographique ${ }^{8}$ au sens où l'a défini Philippe Lejeune pour l'autobiographie. Si certains écrivains refusent de qualifier leur œuvre d'autofiction, peu les qualifient néanmoins de stricte autobiographie ${ }^{9}$. Dans Piv a glevo?, la confusion auteur-narrateur-personnage est entretenue sur la quatrième de couverture alors que la couverture elle-même porte l'indication «romant» [roman]. L'entretien volontaire de cette ambiguité qui passe, notamment, par la mention du terme « $\operatorname{roman}^{10} »$, n'est pas une spécificité de la littérature bretonne : il s'agit, au contraire, d'une tendance bien ancrée dans les littératures contemporaines. Aborder la rhétorique de ces textes, ajoute à la méfiance à l'égard du genre autobiographique : user d'artifices stylistiques afin de mieux se faire comprendre ou de donner plus de force à son propos pourrait éloigner de la sincérité, de la mesure, de la prétendue objectivité de ces discours. Pourtant, tout texte porte nécessairement une part de rhétorique inhérente au passage à l'écrit : la «transsubstantiation» du vécu en mots, dont parle Annie Ernaux ${ }^{11}$.

Piv a glevo ? raconte la vie d'un adolescent à Pordic, près de Saint-Brieuc à la fin des années 1950. Au-delà de la vie quotidienne agitée de ce jeune garçon atteint d'asthme, le narrateur se fait l'écho de la guerre d'Algérie ou, plus précisément, des répercussions de cette guerre en Bretagne. Le deuxième personnage important du roman est le père; l'expression de ses idées politiques jalonne le

6. Serge DoubrovsKy, op. cit., 4e de couverture.

7. Philippe LeJeune, L'autobiographie en France, Paris, Armand Colin, 2003 (1971 pour la première édition), p. 59-62.

8. Philippe LeJEune, Le pacte autobiographique, Paris, Seuil, 1996 (1975 pour la première édition), p. 15.

9. C'est le cas d'Annie Ernaux, notamment. Cf. Annie Ernaux, L'écriture comme un couteau, Entretien avec Frédéric-Yves Jeannet, Paris, Gallimard, 2011 (2002 pour la première édition).

10. Philippe Gasparini, op. cit., p. 209.

11. Annie ERnAUX, op. cit., p. 103. 
texte, tout comme l'évocation de ses activités secrètes : il soutient les réseaux du Front de Libération Nationale (FLN) algérien installés dans la région de Saint-Brieuc. La variété de langue utilisée dans le texte permet immédiatement d'identifier ce roman comme une œuvre récente dans l'histoire littéraire du breton. Or, le choix du breton «littéraire» ou «disparitaire ${ }^{12} »$, n'est pas une exclusive chez Hervé Ar Gall : on dirait, au contraire, qu'il s'emploie, tout au long de son texte, à brouiller les pistes sociolinguistiques. En plus de termes issus du «néo-breton», l'auteur emploie des expressions imagées ou populaires qui éloignent de cette impression initiale. Entre la pseudonorme ${ }^{13}$ et la truculence de la variété populaire, Hervé Ar Gall ne choisit pas mais pioche alternativement dans les deux registres.

L'autre aspect marquant à la lecture du roman réside dans l'emploi d'expressions qui «font écho» dans l'esprit du lecteur qui est également nécessairement francophone. Il s'agit de tropes ou de clichés linguistiques que l'on entend ou que l'on lit, en français, dans la rhétorique politique ou médiatique ${ }^{14}$. Ces formules-échos utilisées par l'auteur n'appartiennent pas toutes à la même variété de breton; elles n'appartiennent même pas toutes spécifiquement au breton car certaines sont des traductions de tournures issues du français. Le texte présente ainsi parfois un décalage énonciatif étonnant comme si l'auteur voulait réunir dans une même bouche, ou sous une même plume, tout ce qui fait le breton et son histoire. Cette énonciation ambivalente passe, d'une part, par l'emploi de tournures issues du registre populaire et, de l'autre, par de nombreux emprunts à la rhétorique politique. Il s'agira de décrire ces modes d'expression contradictoires, afin de comprendre ce que cette ambivalence nous dit de Piv a glevo ? et du message que souhaite transmettre son auteur.

12. Le registre disparitaire est la variété haute de la diglossie, c'est-à-dire le registre employé en situation formelle. Cf. Yves Le Berre et Jean Le Dû, «Parité et disparité. Sphère publique et sphère privée de la parole», La Bretagne linguistique, $\mathrm{n}^{\circ}$ 10, Brest, CRBC-UBO, 1996, p. 7-25.

13. Cf. Jean LE DÛ, «Chez nous, on ne parle pas le vrai breton», Actes du congrès international Che cosa ne pensa oggi Chiaffredo Roux?, Bardonnecchia, ALEPO, Université de Turin, 2002, p. 37-59.

14. Sur les caractéristiques du discours politique, cf. Patrick Charaudeau, "L'imaginaire de la 'souveraineté populaire'», Le discours politique. Les masques du pouvoir, Paris, Vuibert, 2005, p. 175-190. 


\section{La variété populaire}

La figure de rhétorique majoritaire dans le roman est de loin la métaphore : en moyenne, une métaphore par page. Ceci s'explique de diverses manières : la métaphore est, tout d'abord, selon le groupe $\mu^{15}$, «la figure centrale de la rhétorique ${ }^{16} »$. Dans la littérature de langue française du $\mathrm{XX}^{\mathrm{e}}$ siècle, ce sont les surréalistes qui ont développé et poussé au maximum les possibilités offertes par cette figure de style ${ }^{17}$. Ce goût d'Hervé Ar Gall pour la métaphore s'explique aussi par le fait qu'elle est également une figure fréquente dans la langue orale, au même titre que la comparaison ou la litote ${ }^{18}$. Dans le registre paritaire ${ }^{19}$, elle permet de substituer aux termes abstraits, des termes concrets, beaucoup plus frappants pour l'imagination. En breton, la métaphore est considérée par Jules Gros comme l'une des figures préférées de la langue populaire. Dans le premier volume du Trésor du breton parlé ${ }^{20}$ consacré au langage figuré où il fait la liste des tropes qu'il a pu collecter, la métaphore est présentée comme la figure qui donne le plus d'expressivité au discours. Elle est tellement fréquente que le sens métaphorique de certains mots va jusqu'à supplanter leur sens originel dans certains des exemples donnés par Jules Gros. Ils ne sont plus considérés comme des figures métaphoriques et ne sont donc plus identifiés comme des «accidents de l'énonciation ${ }^{21} \gg$ puisqu'ils constituent la manière ordinaire de s'exprimer.

Quand il cherche à définir ce que pourraient être les caractéristiques du breton populaire, Jules Gros établit une liste de critères

15. Le « groupe $\mu$ » est un groupe de chercheurs issus du centre d'études poétiques de l'université de Liège dont les travaux interdisciplinaires, depuis la fin des années 1960, portent sur l'esthétique, la linguistique ou la sémiotique. Cf. GroupE $\mu$, Rhétorique générale, Paris, Seuil, 1982, 4e de couverture.

16. Groupe $\mu$, ibidem, p. 91.

17. Groupe $\mu$, ibid., p. 94.

18. Yves Le Berre, «La force et l'élégance. Les litotes dans la Passion bretonne de $1530 »$, La Bretagne Linguistique, n ${ }^{\circ}$ 16, Brest, CRBC-UBO, 2011, p. 123.

19. Le registre paritaire est la variété basse de la diglossie, c'est-à-dire le registre employé en situation informelle. Cf. Yves LE BERRE et Jean LE Dû, «Parité et disparité. Sphère publique et sphère privée de la parole»,op. cit.

20. Cf. Jules Gros, Le Trésor du breton parlé. Le langage figuré, Brest, Emgleo Breiz, 2010.

21. Cf. Yves Le BerRe, La rhétorique des gwerzioù, dans le présent ouvrage. 
(qu'il ne considère pas comme spécifiques au breton). Selon lui, le «langage populaire» est ainsi défini :

1. il préfère les termes concrets aux termes abstraits,

2. il est subjectif et affectif,

3. il a une tendance à exagérer,

4. il est énergique, catégorique et franc plutôt que courtois et appelle un chat un chat,

5. il est négligent, imprécis, capricieux et volontiers gaulois, familier, simple et bon enfant mais adore le jeu verbal, la plaisanterie, la moquerie, le dénigrement ${ }^{22}$.

D'un point de vue sociolinguistique, cette définition est celle du registre paritaire, nommé «badume ${ }^{23}$ » pour ce qui concerne le breton. La lecture de Piv a glevo ?, laisse à penser qu'Hervé Ar Gall reprend à son compte la définition de Jules Gros et la met en pratique dans son roman afin de donner à la langue employée l'apparence de la langue populaire et donc du registre paritaire.

La métaphore a une double qualité : tout en permettant d'éviter les répétitions, elle donne plus de force à l'expression. Hervé Ar Gall utilise majoritairement des métaphores courantes qui ne sont pas des inventions mais des formules ordinaires de la langue populaire. Certaines d'entre elles sont citées dans le dictionnaire de Jules Gros, notamment. Il est possible de classer les métaphores du roman en deux catégories : d'une part, les métaphores issues du breton quotidien, avec, en particulier, celles ayant un référent animal et, d'autre part, celles qui sont des inventions de l'auteur. Quelques exemples tirés de la première catégorie :

- «mestr an $t i^{24} »$ [le maître de la maison] est utilisé à de nombreuses reprises dans le roman pour nommer le père ;

- le père devient également fréquemment «ar penn tiegez $h^{25} »$, expression que l'on trouve chez Jules Gros pour désigner le chef d'une exploitation agricole ;

22. Jules Gros, op. cit., p. 19.

23. Cf. Yves Le BERRE et Jean LE Dû, «Parité et disparité : sphère publique et sphère privée de la parole», op. cit., p. 16.

24. Hervé AR GALL, op. cit., p. 5 (pour la première occurrence).

25. Hervé AR GALL, op. cit., p. 6 (pour la première occurrence). 
- le sens figuré du mot «klud» [perchoir] est utilisé abondamment dans diverses métaphores : "kas mamm-gozh ganit ez klud ${ }^{26}{ }_{\text {» }}$ au sens de «prends ta poupée pour aller te coucher» ou «digludet out ? ${ }^{27} »$, littéralement «tu es descendu de ton perchoir ? » pour «tu es levé ?»;

- «Kraban, krabanoù ${ }^{28} »$ [griffe(s)], de même que «pav, pavioù» [patte(s)], servent régulièrement à désigner les mains ;

- «beg-e-dok» ou «beg-o-zok ${ }^{29} »$ [bout de son/leur chapeau] pour désigner la gendarmerie ;

- «mic'hiek» [morveux] est également employé à plusieurs reprises pour désigner le narrateur lui-même : «n'on nemet ur mic'hiek diboell c'hoaz $h^{30} \gg$ [je ne suis encore qu'un morveux imbécile].

- Enfin, les épisodes d'ivrognerie de la mère, du père ou du narrateur lui-même sont également l'occasion de se servir des expressions imagées du registre paritaire : «ur sac'had mat a oa gantañ ${ }^{31} »$ [littéralement : il portait un sac plein] ou «ne oan ket bet evit mont d'ar gêr gant an tortad a oa ganin» [je ne pouvais rentrer chez moi avec la charge que j'avais ${ }^{32}$.

Les métaphores construites à partir de références animales sont fréquentes dans le breton populaire. En voici quelques exemples tirés de Piv a glevo? :

- «e zivskoaz marc'h-labour ${ }^{33} »$ [ses épaules de cheval de trait];

- «al loen vil, al loen fall ${ }^{34} »$ [la vilaine bête, la sale bête] pour désigner un enfant difficile, le narrateur lui-même, en l'occurrence ;

26. Hervé AR GALl, op. cit., p. 40. Mamm-gozh est le surnom d'une poupée en costume breton.

27. Hervé Ar GALl, op. cit., p. 46.

28. Hervé AR GALl, op. cit., p. 104.

29. Hervé AR GALl, op. cit., p. 105.

30. Hervé Ar GALL, op. cit., p. 84.

31. Hervé Ar GALl, op. cit., p. 29.

32. Hervé AR GALL, op. cit., p. 154.

33. Hervé AR GALl, op. cit., p. 57. "Marc’h labour» est déjà une métaphore désignant un bourreau de travail.

34. Hervé AR GALl, op. cit., p. 57, p. 59. 
$-\left\langle k_{\text {kozh loened }}^{35} »[\right.$ les vieux animaux] pour nommer les petits bourgeois sortant de la messe ;

- «c'hwiled ar vourc $h^{36} »$ [les coléoptères du bourg] pour qualifier les jeunes trainant au bourg.

La volonté de l'auteur d'ancrer son texte dans le registre paritaire est accentuée par l'emploi de jurons ou d'insultes. Le roman regorge de formules grossières ou de références sexuelles dans les dialogues entre les personnages ${ }^{37}$. L'auteur utilise des jurons fréquents issus de la langue populaire, par exemple : "gast ar c'hast ${ }^{38}$ " ou «gastoù ${ }^{39} »$ [putain], «mallozh Doue ${ }^{40} »$ [nom de Dieu], «kaoc'h $k i^{41} \gg$ [merde] ou pour les insultes : " penn brell ${ }^{42}$, mab gast ${ }^{43}$, loen brein $^{44}$, lustrugenn ${ }^{45} \ldots$ » [imbécile, fils de pute, sale bête, trainée...].

À ces métaphores classiques ainsi qu'aux exclamations et insultes issues du registre paritaire, s'ajoutent des formules inventées par l'auteur qui constituent la deuxième catégorie de métaphores. Ces figures originales sont souvent formées à partir d'un lexique appartenant à un registre bien différent du précédent. Pour identifier le père, l'auteur se sert de la métaphore «penn meuriad ${ }^{46}{ }$ faite sur le même modèle que «penn tiegezh»; sauf que «meuriad» est un néologisme inventé par Jean-François Le Gonidec pour traduire le terme «tribu ${ }^{47} \gg$. L'effet recherché - à savoir, utiliser la métaphore pour donner l'apparence du breton populaire au texte - est toujours

35. Hervé AR GALL, op. cit., p. 31.

36. Hervé AR GALL, op. cit., p. 49.

37. Dans le théâtre classique déjà, les jurons servaient à signaler l'origine populaire des personnages. Cf. Françoise Rubellin, Marivaux dramaturge. La double inconstance. Le jeu de l'amour et du hasard, Paris, Champion, 1996, p. 219.

38. Hervé AR GALL, op. cit., p. 47.

39. Hervé AR GALL, op. cit., p. 14.

40. Hervé AR GALL, op. cit., p. 13.

41. Hervé AR GALL, op. cit., p. 58.

42. Hervé AR GALL, op. cit., p. 36.

43. Hervé AR GALL, op. cit., p. 37.

44. Hervé AR GALL, op. cit., p. 57.

45. Hervé AR GALL, op. cit., p. 70.

46. Hervé Ar Gall, op. cit., p. 9.

47. Jean-François LE GONIDEC, Dictionnaire français-breton de Le Gonidec, SaintBrieuc, Prudhomme, 1850, p. 443. 
le même mais le résultat est bien différent d'un point de vue sociolinguistique. De même, la synecdoque «ar pederrodeg ${ }^{48}$ » [quatreroues] est vraisemblablement une invention de l'auteur et un équivalent évident des «deux-roues» français, adapté à l'automobile.

Parmi les métaphores ou figures de style originales en breton se trouvent aussi des formules tirées directement d'expressions françaises :

- «degas dour d'ar vilin ${ }^{49} »$ [apporter de l'eau au moulin];

- «diskaret e bleuñv o yaouankiz ${ }^{50} »$ [abattu dans la fleur de l'âge];

- «chupennoù du » pour désigner des bandes de jeunes de l'époque, traduction directe du français «blousons noirs».

L'auteur utilise d'autres traductions de métaphores issues du français mais dont la compréhension n'est probablement pas aussi immédiate que le «degas dour d'ar vilin»:

- «lonkañ Asphalt ${ }^{51} »$ [avaler l'asphalt] ;

- «yudadennoù an ambulañs ${ }^{52}{ }$ [ [le hurlement (des sirènes) de l'ambulance];

- «paotred an urzh ${ }^{53} »$ [les hommes de l'ordre] pour désigner les policiers, les gendarmes ou les militaires, comme l'équivalent français des «forces de l'ordre», sans être pour autant une traduction littérale.

Enfin, «ar C'hwec'hkorn ${ }^{54} »$ qui veut dire «Hexagone», ici au sens métaphorique. Cette expression, qui ne s'entend que très peu, voire jamais, s'avère être utilisée dans certains titres de la presse de langue bretonne, dans la revue mensuelle d'information Bremañ, notamment.

48. Hervé Ar GALl, op. cit., p. 9.

49. Hervé Ar GALl, op. cit., p. 81.

50. Hervé Ar GALl, op. cit., p. 26.

51. Hervé AR GALL, op. cit., p. 30.

52. Hervé Ar GALl, op. cit., p. 14.

53. Hervé AR GALL, op. cit., p. 79.

54. Hervé AR GALl, op. cit., p. 115. 
L'auteur utilise également des formules grossières issues, elles aussi, d'expressions existant en français : "gast vorn ${ }^{55}$ » [pute borgne] ou «chaokerien kaoc'h an DST $^{56}$ » [les bouffeurs de merde de la DST], par exemple. Les insultes employées dans le roman ne sont donc pas toutes forcément piochées dans le registre du breton populaire. Il y a là un paradoxe : alors que métaphores, exclamations et insultes servent à donner au texte l'apparence de la langue populaire, l'auteur se sert, pour construire certaines d'entre elles, de mots ou d'expressions issus du registre disparitaire et, par conséquent, de néologismes inconnus de la majorité des bretonnants ou encore d'expressions courantes en français, certes, mais qui traduites littéralement en breton donnent une tonalité surprenante à certains passages du texte.

Hervé Ar Gall profite d'autres occasions pour employer des formules ou expressions ancrant son texte dans le registre paritaire, il s'agit des passages décrivant l'éveil de l'intérêt de l'adolescent pour les jeunes filles et les femmes plus mûres - essentiellement les prostituées - et qui sont l'occasion d'abondants commentaires d'ordre sexuel sur les filles et les femmes que le narrateur et les autres garçons croisent sur leur chemin. Au milieu du roman, par exemple, le narrateur surprend la mère d'un de ses amis avec son amant ${ }^{57}$. Ceci est l'occasion d'une longue description détaillée de leurs ébats - plus de trois pages - en forme d'hypotypose. Cette figure de style qui laisse de côté la vision globale d'une scène pour n'en garder que les aspects les plus frappants pour l'imaginaire est souvent employée dans les romans policiers, fantastiques ou encore érotiques. En se focalisant sur un seul aspect de la scène, l'hypotypose porte une force évocatrice se rapprochant de certaines techniques cinématographiques ${ }^{58}$. Certains passages de Piv a glevo? marquent-ils ainsi une rupture nette avec la majorité de la production littéraire de langue bretonne qui était essentiellement sous influence religieuse, jusqu'au premier quart du $\mathrm{XX}^{\mathrm{e}}$ siècle? S'il est relativement fréquent de trouver ce genre de description dans la littérature de langue française

55. Hervé AR GALL, op. cit., p. 47.

56. Hervé AR GALL, op. cit., p. 76.

57. Hervé AR GALL, op. cit., p. 126 à 129.

58. Cf. Michèle AQUIEN et Georges MoliniÉ, Dictionnaire de rhétorique et de poétique, Paris, Librairie générale française, 1999, p. 195-196. 
grand public ${ }^{59}$, on peut considérer qu'il s'agit d'une thématique pour le moins récente dans une littérature - qui se voudrait - grand public en breton ${ }^{60}$. Il existe pourtant depuis le XVIII ${ }^{\mathrm{e}}$ siècle quelques textes de langue bretonne que l'on a pu qualifier de libertins, satiriques ou scatolotiques ; c'est le cas de l'œuvre de François Pascal de Kerenveyer ${ }^{61}$, par exemple ou de la revue underground des années 1970 : Yod Kerc' $h{ }^{62}$. Une différence importante doit cependant être prise en compte : alors que Le bouffon moqueur ou Yod Kerc'h étaient destinés à un public bien identifié, ce roman prétend s'adresser à tout lecteur de langue bretonne; loin, donc, des initiés, qu'ils soient auditeurs ${ }^{63}$ avertis de la noblesse roscovite ou étudiants amateurs de revues satiriques post soixante-huitardes.

Hervé Ar Gall semble faire le choix de rester proche de la variété populaire du breton, pourtant, on peut difficilement imaginer que son roman puisse être lu par des bretonnants de naissance comme le sont certains articles de journaux, certaines pièces de théâtre ou certains recueils de contes. L'auteur ne se contente pas, en effet, d'utiliser des formules strictement populaires, il détourne ces tropes avec des mots, issus d'un registre différent. Ses formules personnelles

59. Cf. Dominique VIART et Bruno Vercier, La littérature française au présent, Paris, Bordas, 2008, p. 336-342 ou p. 355-357. Les auteurs de l'ouvrage citent par exemple certaines œuvres de Philippe Sollers, Virginie Despentes ou encore Catherine Millet.

60. La première décennie des années 2000 voit l'apparition d'ouvrages en breton qui abordent la thématique sexuelle ou la question du genre, à l'image de ce qui se publie dans d'autres langues : cf. Eve ENSLER, Ar forzhioù a gomz, Lannion, An Alarc'h, 2010. Il s'agit d'une traduction en breton des Monologues du vagin ou encore Yann-Fulup Dupouy, Par dibar, Lannion, An Alarc'h, 2007. Il s'agit d'un roman où le narrateur raconte comment il a pris conscience et assumé son homosexualité. Ces nouveautés en matière de littérature bretonne suivent ce qui s'écrit en littérature de langue française notamment, cf. Dominique VIART et Bruno VERCIER, «Littérature et 'communautés'», La littérature française au présent, op. cit., p. 336 à 345.

61. F.-N. PASCAL DE KERENVEYER, Ar farvel goapaer. Le bouffon moqueur, traduit et présenté par Ronan Calvez, CRBC-UBO, Brest, 2005.

62. Yod Kerc'h est une revue satirique de langue bretonne publiée au milieu des années 70 par des étudiants rennais.

63. Le texte de Kerenveyer avait vocation à être déclamé en public. Cf. Ronan CALVEZ, «Métamorphose de Kerenveyer», dans F.-N. PASCAL DE KerenVeYer, Ar farvel goapaer. Le bouffon moqueur, op. cit., p. 45. 
mélangent souvent des aspects qui paraissent sociolinguistiquement incompatibles : faire des métaphores - et donc donner l'apparence de la langue populaire - mais en employant des néologismes inconnus dans ce registre du breton.

\section{La rhétorique politique}

La langue utilisée dans les passages illustrant l'engagement politique du père comporte peu de métaphores mais d'autres particularités stylistiques permettent de la définir plus précisément : il y a restitution, en breton, de formules issues de la rhétorique d'extrême gauche ou de celle de l'anticolonialisme. Leur particularité n'est plus de faire écho au breton populaire mais de nous «faire entendre» du français derrière les mots bretons utilisés :

Pevar bugel eus Porzhig a zo bet lazhet er vro-hont, diskaret e bleuñv o yaouankiz, evit klod [renom] hag enor ar vamm-vro, hag an drevadennerien. Evit Bro Frañs disrannus ha peurbadus oc'h astenn eus Dukard da dTamanraset... ${ }^{64}$

Ce qui donnerait mot pour mot en français :

Quatre enfants de Pordic ont été tués dans un pays lointain, abattus dans la fleur de l'âge pour le renom et l'honneur de la mère patrie et des colonialistes. Pour la France indivisible et éternelle s'étendant de Dunkerque à Tamanrasset... ${ }^{65}$

«L'honneur de la mère-patrie», «la France indivisible» et bien sûr « de Dunkerque à Tamanrasset» passent pour être des expressions familières chez un lecteur, bretonnant certes, mais aussi nécessairement francophone. La dernière formule est une référence extraite d'un discours du président de Gaulle reprise et détournée à plusieurs occasions, au point de devenir une expression clé du corpus des formules liées à la guerre d'Algérie ${ }^{66}$ et réutilisées par les partisans, comme les opposants, à l'Algérie française. Cet exemple se distingue nettement de la variété de breton décrite précédemment : l'emploi

64. Hervé AR GALL, op. cit., p. 26.

65. Les traductions sont de moi.

66. Cf. Benjamin Stora, Les mots de la guerre d'Algérie, Toulouse, Presses universitaires Toulouse-Le Mirail, 2005. 
des substantifs «klod»[renom] par exemple et «trevadennerien» [colonialistes] ainsi que des adjectifs "disrannus ha peurbadus» [indivisible et éternelle] ne font pas partie de la langue populaire. Le terme «disrannus» est même absent du dictionnaire d'An Here ${ }^{67}$ tout comme de celui de Francis Favereau ${ }^{68}$. L'effet d'écho pousse à le traduire par «indivisible» or, «disrannus» est présent dans le dictionnaire de Roparz Hemon ${ }^{69}$, mais avec le sens de «qui divise», ce qui ne correspond pas au contexte.

Le même effet est présent dans les passages à tonalité politique du roman qui portent l'empreinte des idées d'extrême gauche du père du narrateur. Ainsi, lorsqu'il évoque le responsable de l'accident qui lui a valu de perdre une jambe, son discours adopte une tonalité clairement syndicaliste :

An Aotrou Pinik, Maer Sant Brieg eo ! Penn bras ha mestr an tu dehou en Departamant... Golist kredus. Savet a-du gant De Gaulle (sic) goude an taol-stad a oa bet sevenet gantañ $e$ 1958 ... Ur c'hilstourmer eus ar re washañ, ur patron kriz gant e vicherourien. Stourm a ra didruez ouzh dilennidi ar sindikajoù en embregerez $h^{70}$.

C'est M. Pinic, le maire de Saint-Brieuc. À la tête de la droite dans le département. Gaulliste absolu. Il s'est allié à de Gaulle après le coup d'état qu'il a organisé en 1958 ... Un réactionnaire de la pire espèce, un patron cruel envers ses ouvriers. Il se bat de manière intraitable contre les élus des syndicats dans l'entreprise.

Comme dans le passage précédent au sujet de la guerre d'Algérie, l'auteur fait clairement le choix de la néologie : taol-stad [coup d'État], ur c'hilstourmer [un réactionnaire], embregerezh [entreprise]... tous ces termes indiquent la volonté de constitution, en breton, d'un discours de type syndicaliste basé sur son équivalent en français. Un siècle auparavant, la question des modèles et des influences s'est posée également à Hervé Burel ${ }^{71}$ qui, pour des dis-

67. Geriadur brezhoneg, Le Releq-Kerhuon, An Here, 2001.

68. Francis FAVEREAU, Geriadur ar brezhoneg a-vremañ, Morlaix, Skol Vreizh, 1993.

69. Roparz Hemon, Dictionnaire Breton-Français / Français-Breton, Brest, Al Liamm, 1997.

70. Hervé AR GALL, op. cit., p. 88.

71. Hervé BuREL, Histor eur famill eus Breïs-Izel - Histoire d'une famille de Basse- 
cours à tonalité similaire, a adopté des choix linguistiques différents : ceux de son temps mais aussi ceux d'un bretonnant de naissance.

Le père du narrateur est relativement indépendant d'un point de vue idéologique. Ainsi, après un discours de type syndicaliste, certains passages portent-ils l'empreinte d'une tonalité politique un peu différente, le père faisant entendre un discours tiers-mondiste, qui s'explique par ses activités en faveur du FLN :

Ma zad ne starde ket daouarn paotred an urzh, enebourien ar bobl, mevelien ar Stad hag ar gouarnamant. Ar re-mañ a sikoure ar gevalaourien da lavaret eo an dud o deus arc'hant bras da waskañ ha da gorvoiñ ar vicherourien hag ar pobloù a vank dezhe bevañ dieub ha bezañ mestr en o bro ${ }^{72}$.

Mon père ne serrait pas la main des forces de l'ordre, les ennemis du peuple, les serviteurs de l'État et du gouvernement. Ceux-là aidaient les capitalistes, c'est-à-dire ceux qui ont beaucoup d'argent, à oppresser et exploiter les ouvriers et les peuples qui ne demandent qu'à être libres et maîtres dans leur pays.

Ce passage témoigne soit d'une volonté pédagogique, soit d'une volonté de distinction ${ }^{73}$ de la part de l'auteur: la formule «da lavaret eo» [c'est-à-dire] introduisant la définition du terme "kevalaourien» [capitalistes]. Ce substantif, issu d'une racine galloise, est très certainement inconnu de la majorité des lecteurs, ce que l'auteur anticipe en l'explicitant. Ce terme a été inventé par le SADED ${ }^{74}$ : créé en 1962, son but était de former des néologismes en vue de l'enseignement futur du breton.

Toujours dans cette volonté d'ancrer le roman dans la rhétorique politique, l'exemple suivant s'apparente à une véritable harangue du père :

Bretagne, traduit et présenté par Nelly Blanchard, Brest, CRBC - Morlaix, SkolVreizh, 2011.

72. Hervé AR GALL, op. cit., p. 18.

73. Pierre Bourdieu, La distinction. Critique sociale du jugement, Paris, Les éditions de minuit, 1992 (1979 pour la première édition), p. 216 ou Ce que parler veut dire. L'économie des échanges linguistiques, Paris, Fayard, 2004 (1982 pour la première édition), p. 56, 60, 76 .

74. Strollad an Deskadurezh Eil Derez [Le groupe pour l'enseignement secondaire]. 
N'hellomp ket ni, demokraterien, degemer e vefe boureviet kement studier aljerian ha chom diseblant ouzh seurt darvoudoù, hon dever eo sevel a-du gante hag o skoazellañ en o stourm a-enep an trevadennour hag evit dieubidigezh o bro. Dever an tu kleiz brezhon eo mont en tu all d'al lavar ha degas ur skoazell fetis hag efedus da bobl Aljeria... An tu kleiz gall n'hell ket e ober rak n'eo ket eneptrevadennour. B'emañ, d'am meno, kevarzhelour, da lâret eo : emañ mat d'ar gleizerien-se vefe ingalded ar madoù etre an dud ha b'emaint a-enep korvoiñ an nen war dachenn an ekonomiezh padal emaint impalaerien dre ideologiezh... Guy Mollet, Prezidant Kuzul Bro C'hall, a oa unan eus pennoù emsav micherourien Bro C'hall; n'eo ket un den diboell met a zivout brezel Aljeria e tielfenn an darvoudoù e-giz ma rafe forzh peseurt penn renad [régime] kilstourmer zo... Sokialour eo, sur, hag ivez broadelour gall, trevadennour hag hollveliour dre bouez istor ha sevenadur e vro... ${ }^{75}$

Nous ne pouvons, nous les démocrates, accepter que soient torturés tant d'étudiants algériens et rester indifférents à ce genre d'événements, c'est notre devoir de leur manifester notre soutien et de les aider dans leur combat contre le colonialisme et pour la libération de leur pays. C'est le devoir de la gauche bretonne d'aller au-delà des mots et d'aider concrètement et efficacement le peuple algérien... La gauche française ne peut le faire car elle n'est pas anticolonialiste. Elle est, je crois, égalitariste, c'est-àdire que ces gauchistes sont pour la répartition des richesses et contre l'exploitation de l'homme dans le domaine économique ; en revanche, ils sont impérialistes par idéologie... Guy Mollet, le Président du Conseil en France était l'un des chefs du mouvement ouvrier en France; il n'est pas sot mais au sujet de la guerre d'Algérie, il analyse les événements comme le ferait n'importe quel chef de régime conservateur... Il est socialiste, certainement, et aussi nationaliste français, colonialiste et impérialiste à cause du poids de l'histoire et de la culture de son pays...

Ce passage n'est pas, comme sa tonalité pourrait le laisser croire, un discours devant une assemblée de militants; ce sont les propos du père qui s'adresse à son fils. Il y reproduit pourtant des figures de rhétorique propres au discours politique :

75. Hervé AR GALL, op. cit., p. 148. 
l'emploi du «nous» rassembleur ${ }^{76}$, par exemple, avec la formuleécho «ni, demokraterien» [nous, les démocrates]; de même, l'expression fréquente dans la rhétorique politique mettant en balance les paroles et les actes : «Mont en tu all d'al lavar ha degas ur skoazell fetis hag efedus d'ar bobl aljerian» [aller au-delà des mots et aider concrètement et efficacement le peuple algérien]. À ceci s'ajoute l'emploi de phrases courtes et percutantes alternant avec des phrases argumentatives longues, ainsi que l'utilisation de nombreux termes à charge politique ou historique forte : «demokraterien» [démocrates], «boureviet» [torturé], «trevadennour» [colonisateur], «dieubidigezh» [libération], «eneptrevadennour» [anticolonialiste], «kevarzhelour» [égalitariste], «ingalded» [égalité], «korvoiñ» [exploiter], «impalaerien» [imperialistes], «kilstourmer» [réactionnaire], «sokialour» [socialiste], «hollveliour» [impérialiste]... qui placent clairement ce discours dans la tonalité idéologique anticolonialiste et tiers-mondiste des années 1960-1970. Il est à noter, à titre de comparaison, que l'Union démocratique bretonne (UDB) fait des choix linguistiques différents dans sa revue Pobl Vreizh au milieu des années 1970 et préfère «sossialist» ou «sosialist» à «sokialour» [socialiste], «koloniset» à «trevadennet» [colonisé / colonie] ou encore "sosieteoù » ${ }^{77}$ à «embregerezh» [société, au sens d'entreprise]. De même, à la place de «brezel Aljeria» [la guerre d'Algérie] employé dans le roman, Pobl Vreizh fait le choix de «brezel an Aljeri», forme ordinaire que l'on entend dans la bouche des bretonnants de naissance. Ces différences illustrent une évolution sociolinguistique : Pobl Vreizh, dans les années 1970, essaie de ne pas se couper de la pratique du breton de l'époque, ce qui apparaît dans l'emploi de termes utilisés par la majorité des bretonnants; c'est apparemment moins le cas au début des années 2000 avec l'utilisation de néologismes, qui ne sont pas employés par la majorité des bretonnants, mais qui sont, il est vrai, probablement utilisés par la majorité des enseignants et donc, on peut le supposer, connus de la plupart des élèves issus de l'enseignement bilingue ou immersif.

76. Nelly Blanchard, «Présentation», dans Hervé Burel, Histor eur famill eus Breï-Izel - Histoire d'une famille de Basse-Bretagne, op. cit., p. 20.

77. Pobl Vreizh, n 68, 15 sept.-15 oct. 1975 et n 69, 13 nov.-13 déc. 1975. 
Le souci d'explication de certains termes que l'on pouvait trouver pour le «kevalaourien» [capitalistes] cité précédemment disparaît totalement ici. L'auteur use d'une terminologie difficilement compréhensible pour la majorité des bretonnants de naissance mais sans doute aussi pour les lecteurs habituels d'ouvrages en langue bretonne. C'est grâce à l'écho, c'est-à-dire à la résonance de ce type de passage en français, que les lecteurs pourront sans doute déduire le sens général du discours ainsi que celui d'un certain nombre de mots peu connus et absents des dictionnaires de Favereau et d'An Here : «kevarzhelour» [égalitariste] et «hollveliour» [impérialiste], par exemple.

La syntaxe indique également que le modèle de ce genre de discours est à rechercher du côté de la langue française. L'ordre des mots en breton reproduit exactement celui du français, par exemple : «... met a zivout brezel Aljeria e tielfenn an darvoudoù e-giz ma rafe forzh peseurt penn renad kilstourmer zo», devient mot pour mot en français : «... mais à propos de la guerre d'Algérie, il analyse les événements comme le ferait n'importe quel chef d'État réactionnaire». Le calque syntaxique indique que le modèle adopté par l'auteur est bien celui de la rhétorique politique de langue française. Ainsi, les formules-échos de cette rhétorique viennentelles au secours du lecteur afin de rendre certains passages obscurs plus facilement compréhensibles.

\section{Un syncrétisme linguistique au service d'un syncrétisme idéologique}

À la fin du roman, il y a une longue discussion entre le père et le fils; elle est l'illustration d'un débat qui a agité les milieux militants bretons de gauche et d'extrême gauche au milieu des années $1960^{78}$, après avoir été débattu au moment des luttes anticolonialistes en Égypte, Tunisie, Maroc ou Algérie. Comment peut-on être de gauche et, dans le même temps, revendiquer une autonomie pour la Bretagne? Autrement dit, comment allier la lutte des classes et la lutte

78. Cf. Tudi Kernalegenn, Drapeaux rouges et gwenn-ha-du - L'extrême gauche en Bretagne dans les années 1970, Rennes, Apogée, 2005, p. 101-131. Michel Nicolas, Histoire de la revendication bretonne ou la revanche de la démocratie locale sur le «démocratisme», Spézet, Coop Breizh, 2007, p. 213-219. 
nationaliste. À la naissance de l'UDB, s'est ainsi posée la question de la compatibilité entre la défense des prolétaires - qui n'ont pas de patrie, selon les idées marxistes ${ }^{79}$ - et la lutte pour l'autonomie ou l'indépendance de la Bretagne. Lanig, le narrateur, fait remarquer cette contradiction à son père :

Lavar din, tadig, perak e kemerez perzh er stourmadegoùse? Dieubidigezh ar bobl aljerian n'eo ket stourm ar broleterien a-enep renkoù uhellañ ar gevredigezh, perc'henned an industriezh, ar gapitalourien, met kentoc'h stourm ur bobl evit dieubiñ he bro ha kaout kement gwir a zo ezhomm evit sevel ur voad; n'eo ket ur stourm da gas war-raok dre ret etreboadelezh ar broleterien?

Peseurt diskourioù zo ganit, Lanig! Soñjal a ra din me e rank pep pobl bezañ mestr en he bro, ren he dazont ha skarzhañ kuit ar mac'homer hag armeoù ar Stad vac'homer. Ar Stad c'hall eo an alouberez, ha ni, Bretoned, kenkoulz ha pobloù Aljeria hon do ivez da gas war-raok ur stourm dieubiñ hor bro evit adkavout hor gwirioù ha sevel ur vroad e Breizh. Proleterien Breizh a c'hello kemer perzh e stourmoù etrebroadel evel Bretoned mestr en o bro.

Dis-moi, papa, pourquoi tu participes à ces luttes ? La libération du peuple algérien n'est pas la lutte des prolétaires contre les classes supérieures de la société, les patrons d'industrie, les capitalistes mais plutôt la lutte d'un peuple pour libérer son pays et obtenir les doits nécessaires pour créer une nation ; n'est-ce pas la lutte pour l'internationalisme prolétarien qu'il faut mener en priorité ?

Quels discours tu tiens, Lanig! Je pense moi que chaque peuple doit être maitre en son pays, diriger son avenir, expulser les oppresseurs et les armées de l'État oppresseur. L'État français est l'envahisseur, et nous, Bretons, tout comme les peuples d'Algérie, devrons aussi mener une lutte pour la libération de notre pays, retrouver nos droits et créer une nation en Bretagne. Les prolétaires bretons pourront participer aux luttes internationales comme des Bretons maîtres dans leur pays.

79. Karl MarX et Friedrich Engels, Manifeste du parti communiste, Paris, AubierMontaigne, 1971, p. 121. 
Ce passage met en lumière un dernier aspect de l'idéologie paternelle qui mêle communisme, syndicalisme et nationalisme breton : le père exprime dans le passage qui précède la position de l'UDB des années 1960-1970 qui va s'atteler à prouver qu'il n'est pas contradictoire de lier les revendications pour l'autonomie de la Bretagne et la lutte des classes. La défense et illustration de cette position était expliquée dans une brochure de l'UDB publiée en 1972, pour justifier la création de ce nouveau parti politique et qui porte le titre explicite suivant : Bretagne $=$ colonie $^{80}$.

La rhétorique ambivalente de Piv a glevo? peut être considérée comme une illustration de la volonté de réunir deux formes de lutte politique qui peuvent être vues comme idéologiquement difficilement conciliables. La coexistence dans le roman d'éléments sociolinguistiquement très divers peut ainsi être interprétée politiquement. Le peuple serait représenté dans l'œuvre par le registre paritaire du breton, se manifestant par l'emploi de métaphores, de jurons ou de grossièretés issues de la langue populaire. Il s'agit, par la rhétorique, de manifester l'ancrage populaire de la langue bretonne, celle des bretonnants. En revanche, dans les passages concernant la politique, c'est clairement la pseudo-norme qui est mise en avant, c'est-àdire la norme sans État qui use de nombreux néologismes mais qui est également d'une structure syntaxique très proche de celle de la langue française, autrement dit le «britton $»{ }^{81}$. Il s'agirait de la norme d'une future Bretagne autonome.

Le syncrétisme linguistique d'Hervé Ar Gall est donc au service d'un syncrétisme idéologique : réunir, dans une même œuvre, des éléments linguistiques d'origine très différente, comme pour prouver qu'ils ne sont pas contradictoires. Au point de vue linguistique, Piv a glevo ? fait fusionner la langue populaire et ce que serait la future norme nationale du breton, tout comme il fait fusionner, au point de vue idéologique, la défense du prolétariat et celle de la Bretagne comme future entité politique distincte de la France. C'est ce que

80. Union démocratique bretonne, Bretagne=colonie, Avec l'UDB pour que ça cesse !, Rennes, s. n., 1964.

81. Ronan CALvEZ, «Ce que parler du breton veut dire», Ethnologie française, vol. 42, 2012, p. 652-653. 
suggère le slogan - très rhétorique - de l'UDB des débuts : «Kleiz Breizh $^{82} »$ [Bretagne à gauche].

En 1925, Roparz Hemon posait avec Gwalarn ${ }^{83}$ les bases d'une nouvelle langue écrite rigoureusement unifiée grammaticalement, dialectalement et, plus tard, orthographiquement ${ }^{84}$. Il était dans l'exclusive; Hervé Ar Gall, quant à lui, rechercherait de la fusion. Il ne s'agit plus d'évacuer une partie de l'histoire de la langue et de ses locuteurs mais de prouver la capacité de la langue bretonne à tout dire et à adopter tous les registres. Ceci permet de chercher à rattacher aux wagons de la future nation autonome, ce(ux) qui avai(en)t été oublié(s) ou exclu(s) par Hemon, à savoir la dimension populaire et paritaire du breton, et, par là même, la majorité des bretonnants. La réunion, dans un même texte, de traits sociolinguistiquement contradictoires pourrait être interprétée comme une méconnaissance de l'histoire sociolinguistique du breton ou comme la négation de celle-ci. Il s'agirait ici, au contraire, de la mise en pratique linguistique d'un discours politique : prouver que la langue bretonne peut garder la place qu'elle occupe déjà, à savoir la langue paritaire d'une partie de la population, tout en montrant qu'elle peut devenir également une future norme nationale. La fusion linguistique est à l'image de la fusion politique recherchée par l'UDB dans les années 1960-1970 qui plaçait revendication sociale et revendication nationale sur le même plan $^{85}$.

82. Michel NicOlas, op. cit., p. 186.

83. Roparz Hemon et Olivier Mordrelle, «Premier et dernier manifeste de Gwalarn en langue française», Breiz Atao, mars 1925.

84. Cf. Ronan CALVEZ, «1941 : le breton, langue d'État», dans Christian BougEARD (dir.), Bretagne et identités régionales pendant la Seconde Guerre mondiale, actes du colloque international (15-17 novembre 2001), Brest, CRBC-UBO, 2002, p. 207-221.

85. Union démocratique bretonne, Notre parti : l'union démocratique bretonne, s.l., s.n., 1977, p. 6 ou Jean-Jacques MonNiER, Histoire de l'union démocratique bretonne, s.l., Presses populaires de Bretagne, s.d., p. 5. 


\section{Bibliographie}

AQUIEN Michèle et MoLINIÉ Georges, Dictionnaire de rhétorique et de poétique, Paris, Librairie générale française, 1999.

BouRdiEu Pierre, Ce que parler veut dire. L'économie des échanges linguistiques, Paris, Fayard, 2004 (1982 pour la première édition).

BOURDIEU PIERRE, La distinction. Critique sociale du jugement, Paris, Les éditions de minuit, 1992 (1979 pour la première édition).

BUREL Hervé, Histor eur famill eus Breïs-Izel - Histoire d'une famille de Basse-Bretagne, traduit et présenté par Nelly Blanchard, Brest, CRBC - Morlaix, Skol-Vreizh, 2011.

CALVEZ Ronan, «1941 : le breton, langue d'État», dans Christian BOUGEARD (dir.), Bretagne et identités régionales pendant la Seconde Guerre mondiale, actes du colloque international (15-17 novembre 2001), Brest, CRBC-UBO, 2002.

CALvez Ronan, «Ce que parler du breton veut dire», Ethnologie française, vol. 42, 2012.

ChARAUDEAU Patrick, "L'imaginaire de la 'souveraineté populaire'», Le discours politique. Les masques du pouvoir, Paris, Vuibert, 2005.

Doubrovsky Serge, Fils, Paris, Galilée, 1977.

DuPOUY Yann-Fulup, Par dibar, Lannion, An Alarc'h, 2007.

ENSLER Eve, Ar forzhioù a gomz, Lannion, An Alarc'h, 2010.

ERNAUX Annie, L'écriture comme un couteau, Entretien avec FrédéricYves Jeannet, Paris, Gallimard, 2011 (2002 pour la première édition).

FAVEREAU Francis, Geriadur ar brezhoneg a-vremañ, Morlaix, Skol Vreizh, 1993.

GASPARINI Philippe, Autofiction. Une aventure du langage, Paris, Seuil, 2008.

Geriadur brezhoneg, Le Relecq-Kerhuon, An Here, 2001.

Gros Jules, Le Trésor du breton parlé. Le langage figuré, Brest, Emgleo Breiz, 2010.

GROUPE M, Rhétorique générale, Paris, Seuil, 1982.

HEMON Roparz et MORDRELLE Olivier, «Premier et dernier manifeste de Gwalarn en langue française», Breiz Atao, mars 1925.

HEMON Roparz, Dictionnaire Breton-Français / Français-Breton, Brest, Al Liamm, 1997.

KERNALEGENN Tudi, Drapeaux rouges et gwenn-ha-du - L'extrême gauche en Bretagne dans les années 1970, Rennes, Apogée, 2005. 
LE BERre Yves et LE DÛ Jean, «Parité et disparité. Sphère publique et sphère privée de la parole», La Bretagne linguistique, $\mathrm{n}^{\circ} 10$, Brest, CRBC-UBO, 1996.

LE BerRe Yves, «La force et l'élégance. Les litotes dans la Passion bretonne de $1530 »$, La Bretagne Linguistique, $\mathrm{n}^{\circ}$ 16, Brest, CRBCUBO, 2011.

LE DÛ Jean, «Chez nous, on ne parle pas le vrai breton», Actes $d u$ congrès international Che cosa ne pensa oggi Chiaffredo Roux?, Bardonnecchia, ALEPO, Université de Turin, 2002, p. 37-59.

LE GONIDEC Jean-François, Dictionnaire français-breton de Le Gonidec, Saint-Brieuc, Prudhomme, 1850.

LEJEUnE Philippe, L'autobiographie en France, Paris, Armand Colin, 2003 (1971 pour la première édition).

LEJEUNE Philippe, Le pacte autobiographique, Paris, Seuil, 1996 (1975 pour la première édition).

MARX Karl et ENGELS Friedrich, Manifeste du parti communiste, Paris, Aubier-Montaigne, 1971.

MONNIER Jean-Jacques, Histoire de l'union démocratique bretonne, s.l., Presses populaires de Bretagne, s.d.

NICOLAS Michel, Histoire de la revendication bretonne ou la revanche de la démocratie locale sur le "démocratisme», Spézet, Coop Breizh, 2007.

PASCAL DE KERENVEYER François-Nicolas, Ar farvel goapaer. Le bouffon moqueur, traduit et présenté par Ronan Calvez, CRBC UBO, Brest, 2005.

RUBELLIN Françoise, Marivaux dramaturge. La double inconstance. Le jeu de l'amour et du hasard, Paris, Champion, 1996.

STORA Benjamin, Les mots de la guerre d'Algérie, Toulouse, Presses universitaires Toulouse-Le Mirail, 2005.

Union démocratique bretonne, Notre parti : l'union démocratique bretonne, s.l., s.n., 1977.

VIART Dominique et VERCIER Bruno, La littérature française au présent, Paris, Bordas, 2008, 